\title{
Quasi-parallel whistler mode waves observed by THEMIS during near-earth dipolarizations
}

\author{
O. Le Contel ${ }^{1}$, A. Roux ${ }^{1}$, C. Jacquey ${ }^{2}$, P. Robert ${ }^{1}$, M. Berthomier ${ }^{1}$, T. Chust ${ }^{1}$, B. Grison ${ }^{1}$, V. Angelopoulos ${ }^{3}$, \\ D. Sibeck ${ }^{4}$, C. C. Chaston ${ }^{5}$, C. M. Cully ${ }^{6}$, B. Ergun $^{6}$, K.-H. Glassmeier ${ }^{7}$, U. Auster ${ }^{7}$, J. McFadden ${ }^{5}$, C. Carlson ${ }^{5}$,

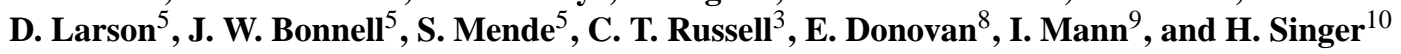 \\ ${ }^{1}$ Laboratoire de Physique des Plasmas, CNRS/Ecole Polytechnique/UPMC/P11, Vélizy, France \\ ${ }^{2}$ Centre d'Etude Spatiales des Rayonnements, CNRS, Toulouse, France \\ ${ }^{3}$ Institute of Geophysics and Planetary Physics, Department of Earth and Space Sciences, University of California, Los \\ Angeles, CA, USA \\ ${ }^{4}$ Goddard Space Flight Center, NASA, Greenbelt, USA \\ ${ }^{5}$ Space Science Laboratory, University of California, Berkeley, CA, USA \\ ${ }^{6}$ Laboratory for Atmospheric and Space Physics, Colorado University, Boulder, USA \\ ${ }^{7}$ Institut für Geophysik und extraterrestrische Physik der Technischen Universität Braunschweig, Braunschweig, Germany \\ ${ }^{8}$ Department of Physics and Astrophysics, University of Calgary, Calgary, Canada \\ ${ }^{9}$ Department of Physics, University of Alberta, Edmonton, Canada \\ ${ }^{10}$ National Oceanic and Atmospheric Administration, Space Environment Center, Boulder, CO, USA
}

Received: 20 October 2008 - Revised: 11 May 2009 - Accepted: 13 May 2009 - Published: 8 June 2009

\begin{abstract}
We report on quasi-parallel whistler emissions detected by the near-earth satellites of the THEMIS mission before, during, and after local dipolarization. These emissions are associated with an electron temperature anisotropy $\alpha=T_{\perp e} / T_{\| e}>1$ consistent with the linear theory of whistler mode anisotropy instability. When the whistler mode emissions are observed the measured electron anisotropy varies inversely with $\beta_{\| e}$ (the ratio of the electron parallel pressure to the magnetic pressure) as predicted by Gary and Wang (1996). Narrow band whistler emissions correspond to the small $\alpha$ existing before dipolarization whereas the broad band emissions correspond to large $\alpha$ observed during and after dipolarization. The energy in the whistler mode is leaving the current sheet and is propagating along the background magnetic field, towards the Earth. A simple timeindependent description based on the Liouville's theorem indicates that the electron temperature anisotropy decreases with the distance along the magnetic field from the equator. Once this variation of $\alpha$ is taken into account, the linear theory predicts an equatorial origin for the whistler mode. The linear theory is also consistent with the observed bandwidth of wave emissions. Yet, the anisotropy required to be fully
\end{abstract}

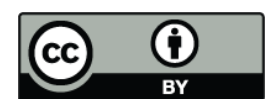

Correspondence to: O. Le Contel (olivier.lecontel@lpp.polytechnique.fr) consistent with the observations is somewhat larger than the measured one. Although the discrepancy remains within the instrumental error bars, this could be due to time-dependent effects which have been neglected. The possible role of the whistler waves in the substorm process is discussed.

Keywords. Magnetospheric physics (Magnetotail; Plasma waves and instabilities; Storms and substorms)

\section{Introduction}

The THEMIS mission is dedicated to the global study of substorms and especially to determine the onset location and the way of propagation of substorms (Angelopoulos, 2008a). However the comprehensive set of instruments mounted onboard each probe allows micro-physics studies too. In the present paper we focus on electromagnetic waves in the whistler frequency range $\Omega_{i}<\omega<\left|\Omega_{e}\right|, \Omega_{i}$ (resp. $\Omega_{e}$ ) being the ion (resp. electron) cyclotron frequency, detected during near-earth dipolarizations.

Whistler mode wave emissions have been observed for a long time in the magnetotail but they were not related to substorm observations. From OGO 1 observations gathered in the near-earth tail $\left(R<17 R_{E}, R_{E}\right.$ means earth radius) Russell (1972) reported on brief bursts of whistler mode magnetic noise close to the neutral sheet. Later from

Published by Copernicus Publications on behalf of the European Geosciences Union. 
IMP 8 observations from 21.1 to $46.3 R_{E}$ in the magnetotail Gurnett et al. (1976) showed that whistler mode magnetic bursts are nearly monochromatic tones lasting from a few seconds to a few tens of seconds with amplitudes of about $100 \mathrm{pT}$ and frequencies from 10 to $300 \mathrm{~Hz}$. They suggested that these emissions could be produced by a current-driven plasma instability. Magnetic bursts were also observed by ISEE 3 in association with plasma sheet crossings by Scarf et al. (1984). Kennel et al. (1986) reported intense bands of whistler mode noise extending up to $\left|\Omega_{e}\right| / 2$ associated with a flux rope signature. They mentioned that the whistler mode noise may require highly anisotropic pitch-angle distributions. More recently from Geotail observations in the region where $-210<X<-10 R_{E}$ Zhang et al. (1999) reported short-lived and narrow band whistler wave emissions propagating in a direction quasi-parallel to the background magnetic field with an average angle of $23^{\circ}$. Their frequencies range between 0.05 to $0.5\left|\Omega_{e}\right|$ with an average value of $0.21\left|\Omega_{e}\right|$. Their amplitude are from few pT to $100 \mathrm{pT}$ with an average value of $44 \mathrm{pT}$. They suggested that whistler waves emissions are very likely to be excited by an energetic electron beam via electron cyclotron resonance. Indeed they pointed out that an electron temperature anisotropy source would provide a whistler emission in both parallel and antiparallel direction to the ambient magnetic field which was not observed. Finally from recent Cluster observations at about $X \simeq-18 R_{E}$ Le Contel et al. (2006) reported broad band whistler wave emissions with very large amplitudes, $(\simeq 1 \mathrm{nT}$ ) observed during a substorm period. These intense emissions last only a few seconds and are associated with very thin current sheets ( $\leq \rho_{i}$, the proton Larmor radius). The whistler mode waves propagated in a direction quasi-parallel to the ambient magnetic field and they occured in conjunction with accelerated electrons. Wei et al. (2007) also gave evidence for whistler mode emissions associated with substorm activity in the magnetotail. Notably weak whistler waves were detected prior to a local southward turning of the magnetic field in a tailward flow. They related the observed enhancement of the whistler mode waves to the development of a collisionless (Hall) magnetic reconnection event. Indeed in the Hall magnetic reconnection model, ions are thought to decouple from the magnetic field (therefore from the electrons which remain attached to the magnetic field) due to the effect of the Hall term in the Ohm's law (Birn et al., 2001), which becomes important at a spatial scale smaller than the ion inertia length $\left(c / \omega_{p i}, c\right.$ being the velocity of light and $\omega_{p i}$ being the ion plasma frequency). In this ion diffusion region, the plasma dynamics is expected to be controlled by whistler mode waves (Mandt et al., 1994) in the case of no guide field or by kinetic Alfvén waves in presence of a guide field (Rogers et al., 2001). Therefore in Hall reconnection models whistler mode waves are expected to be observed in the mid-tail region (between 15-30 $R_{E}$ ) where an X-line configuration is assumed to be created by a tearing mode like instability.
While the possibility of a tearing mode instability in a realistic magnetotail geometry (i.e. with a normal component $B_{z}$ which closes the magnetic field lines) is still a matter of debate (Pellat et al., 1991; Sitnov et al., 1998), in a purely anti-parallel magnetic configuration (with no $B_{z}$ ), the sensitivity of the tearing mode to other parameters has been studied. In particular the role of electron temperature anisotropy was investigated (Karimabadi et al., 2004b). From a threedimensional (3-D) study they found that two instabilities with the wave vector along the current can affect the tearing modes by modifying the saturation level, the growth rate or the unstable spatial scale: (1) The Weibel instability which is driven by the electron anisotropy $\alpha \equiv T_{\perp e} / T_{\| e}<1$ (where $e$ denotes electrons and the other subscripts correspond to direction relative to the background magnetic field $\mathbf{B}_{0}$ ) can increase the single island tearing saturation amplitude up to the singular layer thickness. Such an anisotropy $(\alpha<1)$ is produced by the tearing mode which leads to preferential heating of electrons in the parallel direction. (2) The nonlinear evolution of the lower-hybrid drift instability (LHDI) generates an electron temperature anisotropy $\alpha>1$ within the current sheet (Daughton et al., 2004) which increases the growth rate of the tearing mode and extends the spectrum to very short wavelength. Karimabadi et al. (2004b), however, indicated that whistler anisotropy (WAI) and electron mirror instabilities are destabilized when $\alpha>1$. Thus electrons could be isotropized before the tearing mode grows. Finally Gary and Karimabadi (2006) clarified the respective properties of these three possible growing modes (whistler, mirror and Weibel). While the WAI has a maximum growth rate for a parallel propagation and a real frequency larger than the proton cyclotron frequency, the electron mirror instability corresponds to oblique propagation and zero real frequency. For an unmagnetized plasma and the same electron anisotropy the third one, the Weibel instability, may be excited with a zero real frequency and a maximum growth rate in the direction of the lowest temperature. The WAI was found to have a larger growth rate for a smaller anisotropy than for the electron mirror instability. Thus the minimum value of the electron anisotropy for which the WAI can grow should be considered as an upper bound of the electron anisotropy (assuming that the electron diffusion by whistler waves is faster than the tearing growth which is very likely) and therefore whistler mode waves should limit the expected enhancement of the tearing growth rate associated with the electron thermal anisotropy. More generally, whatever the location in the magnetotail or the substorm model, whistler mode waves could play an important role by controlling the level of electron anisotropy which could be crucial for instabilities at lower frequency (tearing, kink, ...).

Whistler waves emissions were also reported to occur in association with reconnection signatures in the dayside, close to the magnetopause (Deng and Matsumoto, 2001; Drake et al., 1997). More recently Stenberg et al. (2005) observed broad band whistler mode emissions close to the 
THEMIS positions on 2008-01-29/00:00:00.0 from 02:00:00 to 03:50:00

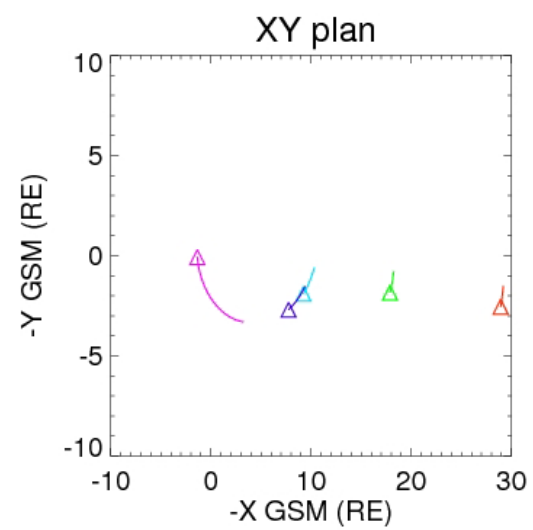

THEMIS-P1 (THB)

THEMIS-P2 (THC)

THEMIS-P3 (THD)

THEMIS-P4 (THE)

THEMIS-P5 (THA)
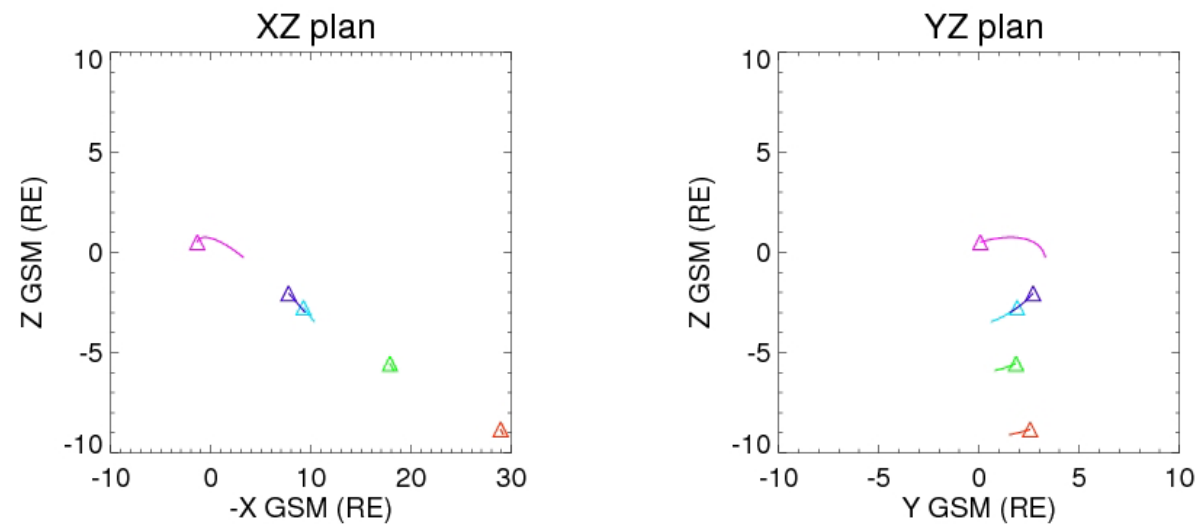

Fig. 1. The locations of the THEMIS probes on the different GSM planes on 29 January 2008. The starting time at 02:00 UT and their trajectories between 02:00 and 03:50 UT are indicated by a triangle and a curve, respectively.

magnetopause, generated in an electron scale current sheet. These emissions were found to propagate in a direction antiparallel to the background magnetic field (away from earth and towards the magnetopause). Stenberg et al. (2005) suggested that the propagation of whistler mode waves only in a direction anti-parallel to $\mathbf{B}_{0}$ is due to the asymmetry, with respect to $v_{\|}=0$, observed simultaneously in the electron distribution.Indeed the electron space phase densities were found to be lower in the parallel than in perpendicular and antiparallel directions; this asymmetry produces a directional anisotropy along $\mathbf{B}_{0}$. Thus whistler mode waves propagating anti-parallel to the ambient field resonate with this directional anisotropy, and therefore undergo amplification, while those propagating along $\mathbf{B}_{0}$ are not amplified because they resonate with isotropic electrons.

The plan of the paper is as follows. Section 2 presents recent THEMIS observations of pseudo-substorm/substorm events on 29 January 2008 between 02:00 and 03:50 UT and focuses on whistler wave emissions. In Sect. 3 we consider the electron temperature anisotropy as a possible mechanism to generate whistler waves and investigate the possible roles these waves can play in the substorm process. We draw conclusions in Sect. 4.

\section{Observations}

\subsection{Global view}

On 29 January 2008 the THEMIS probes began to be lined up along the tail axis during the period between 02:00 and 03:50 UT. Their locations are shown in Fig. 1 in GSM coordinates. Figure 2 displays the magnetic field from the FGM instrument (Auster et al., 2008), the ion density and velocity from ESA (McFadden et al., 2008) gathered during a fast survey period with a time resolution of $3 \mathrm{~s}$. Four dipolarizations can be seen on the $B_{z}$ traces of the two near-earth probes THD (cyan) and THE (blue) at 02:25, 02:38, 02:52, and 03:06 UT while the mid-tail probes THC and THB, which are 


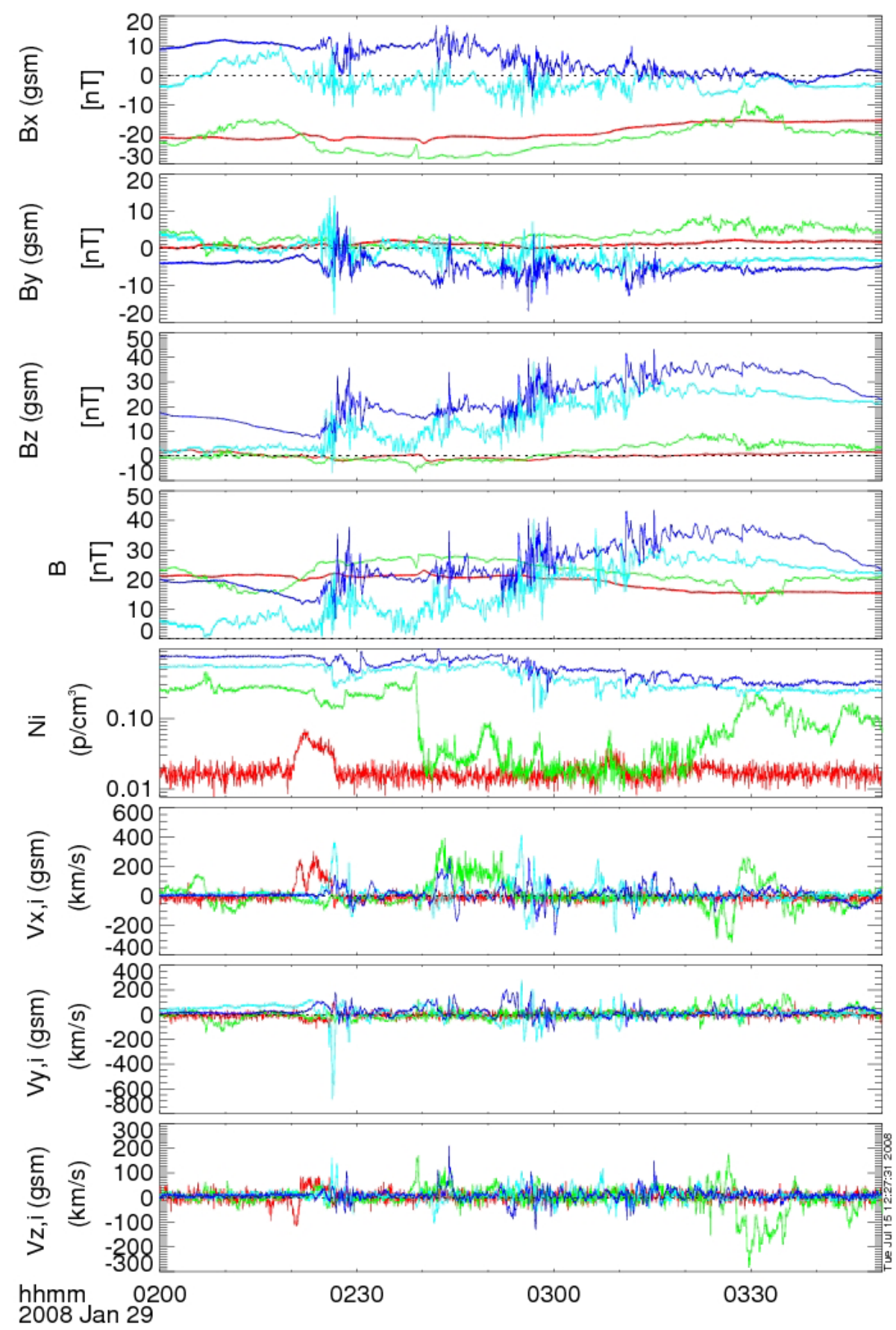

Fig. 2. From top to bottom: Three components and modulus of the magnetic field, Ion density and three components of the ion velocity for four of the THEMIS (traces from THA not displayed). Same color code as for Fig. 1.

located far from the magnetic equator, only measure small magnetic field perturbations. At the beginning of the period THA is still located at the dayside therefore the corresponding curves are not plotted on this figure. An increase of the ion density on THB (red) is seen just before the first dipolarization indicating a vertical (southward) motion or a thicken- ing of the current sheet which is associated with an earthward ion velocity $\left(V_{x}>0\right)$. The second and third dipolarizations are accompanied by a sudden decrease of the ion density measured by THC indicating a vertical (northward) motion or a thinning of the current sheet closer to the Earth which is also associated with an earthward ion velocity; THB farther 


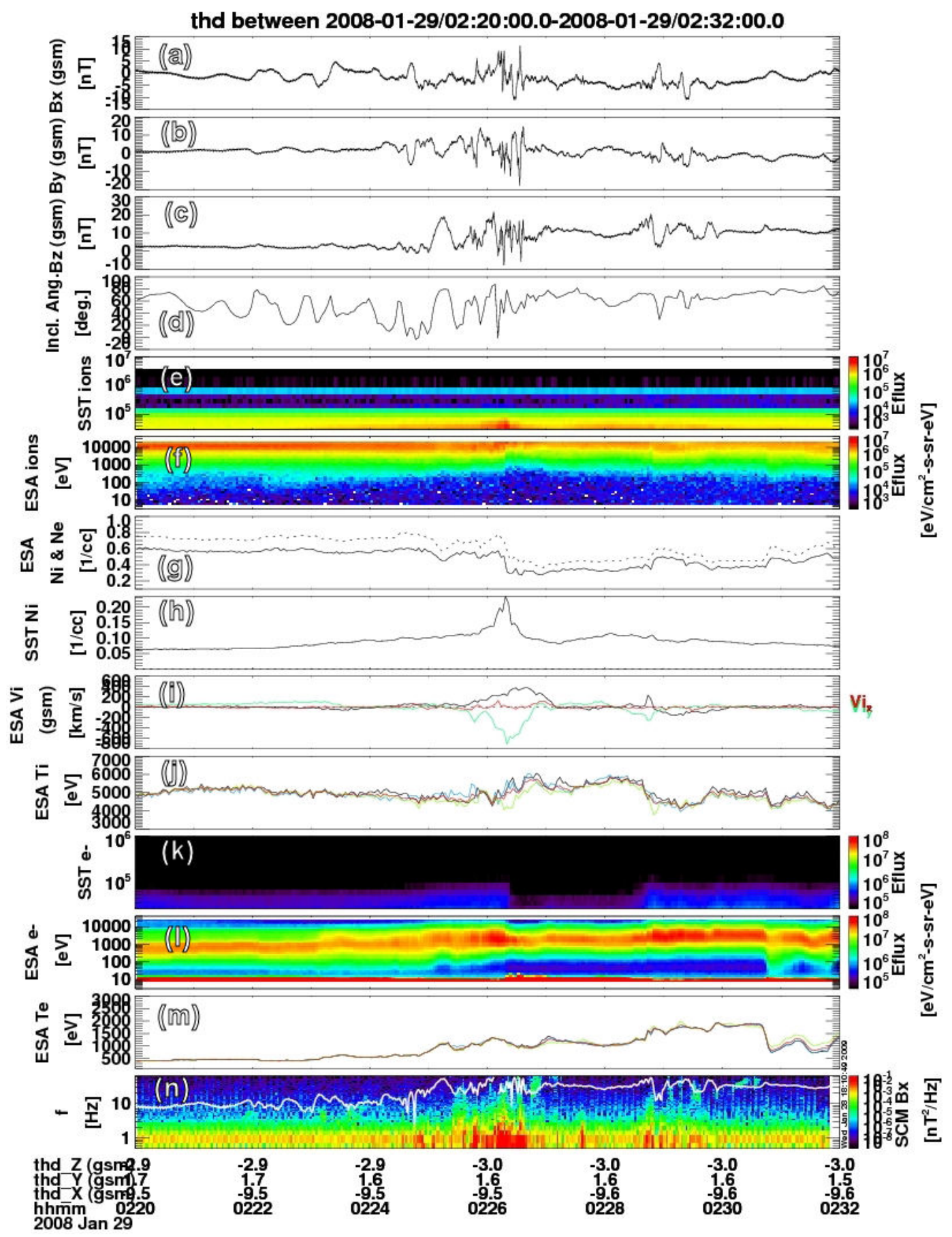

Fig. 3. Panels (a, b, c, d) three components $(128 \mathrm{~S} / \mathrm{s})$ and inclination angle $(3 \mathrm{~s})$ of the magnetic field in GSM coordinates, (e) ion energy spectra from SST and (f) ESA (3 s), (g) ion (solid line) and electron (dotted line) densities from ESA, (h) ion density from SST, (i) ion velocity from ESA in GSM coordinates (Vx:black, Vy:green, Vz:red), (j) ion temperatures from ESA (Txx:black,Tyy:green,Tzz:red,Tav:blue, the averaged temperature Tav=(Txx+Tyy+Tzz)/3), (k) electron energy spectra from SST and (l) ESA, (m) electron temperatures from ESA (same color code as ions), (n) $B_{x}$ power spectral density from SCM ( $2 \mathrm{~s}$ ) from 0.45 to $64 \mathrm{~Hz}$ in GSM coordinates. Superimposed white curve corresponds to $0.1\left|\Omega_{e}\right| /(2 \pi)$. 
from the Earth does not detect any strong density perturbations. For the fourth dipolarization both THC and THB measure a sudden increase of the ion density but without large ion velocity.

There are no large changes of the solar wind pressure or velocity during the 01:00-03:00 UT time period (according to ACE data from CDAWeb, not shown). The ground based observations indicate a substorm activity between $02: 30$ and 04:00 UT as the AE index reaches 400 nT (according to the quicklook Kyoto AE monitor, not shown). A strong decrease of the $\mathrm{H}$ component of the magnetic field is recorded at about 03:00 UT by the Rankin Inlet station (RANK; corrected geomagnetic cordinates $72.3 \mathrm{~N}, 335.8 \mathrm{E}$ ), from the CARISMA array (www.carisma.ca),of the North-America ground based network of magnetometers which covers a sector from 17:00 to 21:00 MLT. Finally a dipolarization is also detected at about 02:57 UT by the geosynchronous spacecraft GOES-12. Thus the first two dipolarizations are likely to be pseudosubstorm breakups while the third and fourth would be two intensifications of the same major substorm event.

\subsection{Near-earth tail observations}

During fast survey periods the THEMIS mission has the capability of capturing bursts of higher time resolution field and particle data. In the magnetotail these burst mode periods are triggered onboard by the fast variations of the z-component of the magnetic field, associated with local dipolarizations (Angelopoulos, 2008a). We now focus on the first dipolarization detected by THD thanks to the onboard burst mode triggering. This dipolarization occurs while the magnetotail is quiet therefore the signatures associated with the dipolarization are clearer. However most of the following observations are also detected by THE for this first event and by THD and THE for the three other events, including the major substorm.

Figure 3 displays an overview of THD observations between 02:20 and 02:32 UT, when the probe was located at $X \simeq-9.5 R_{E}$. Until $\simeq 02: 25: 40 \mathrm{UT}$, the $\left|B_{x}\right|$ component tends to increase while some small oscillations of the current sheet are present (panel a) indicating a thinning of the current sheet. Note that at the beginning of the event THD is located very close to the magnetic equator as the modulus of $B_{x}$ is smaller than $5 \mathrm{nT}$. Then large amplitude magnetic field variations are detected together with an increase, in average, of $B_{z}$ from less than $5 \mathrm{nT}$ to $10 \mathrm{nT}$ around 02:27 UT (panels a, $\mathrm{b}$ and c). A second train of magnetic field variations occurs around 02:30 UT but without changing the background magnetic field; the bipolar signature on $B_{z}$ suggests a flux-rope like structure moving tailward though the $\mathrm{x}$-component of the ion velocity changes from positive to negative at the same time. The inclination angle is plotted on panel $\mathrm{d}$ to emphasize the dipolarization process; its value goes from $60-80^{\circ}$ to $10^{\circ}$ at the end of the substorm growth phase then increases again up to $80^{\circ}$. During the dipolarization ions and electrons are accelerated as the flux of energetic particles around $100 \mathrm{keV}$ measured by SST increases (panels e, $\mathrm{f}, \mathrm{k}$ and $\mathrm{l}$ ). Ions and electrons are also heated from 5 to $6 \mathrm{keV}$ and from $500 \mathrm{eV}$ to $1 \mathrm{keV}$ respectively (panels $\mathrm{j}$ and $\mathrm{m}$ ). Ion and electron densities measured by ESA decrease suddenly at 02:26:20 UT (panel g) but seem to be compensated by the increase of the density of energetic particles measured from SST (panel h). Yet, soon after the densities measured from both experiments decrease. At the same time the y-component of the ion velocity reaches $-700 \mathrm{~km} / \mathrm{s}$ whereas the $\mathrm{x}$-component is about $400 \mathrm{~km} / \mathrm{s}$ toward the Earth. Such a negative $V_{y}$ is difficult to reconcile with the positive y-component of the cross tail current. It implies that electrons also have a negative $V_{y}$, larger in absolute value and are therefore the main current carriers in the spacecraft frame. It has been shown theoretically that a thin current sheet with a spatial scale of the order of the ion Larmor radius may have this property (Sitnov et al., 2000; Schindler and Birn, 2002) and has been already observed by Geotail (Asano et al., 2004) and by Cluster (Baumjohann et al., 2007) farther in the magnetotail. Note that the increases of the density and the fluxes of energetic ions measured by SST imply that the ESA velocities are likely to be underestimated during this period (McFadden et al., 2008). Finally an increase in the magnetic component of the waves is also measured up to the upper limit of $64 \mathrm{~Hz}$ and around $0.1\left|\Omega_{e}\right|$ (plotted as a white curve on panel n) by SCM (Roux et al., 2008; Le Contel et al., 2008) with broad band and narrow emissions in the whistler frequency range (panel $n$ ).

Thus the increase, in average, of the $B_{z}$ component during the time period between 02:22 and 02:28 UT is associated with an increase of the electron and ion temperatures and a decrease of the electron and ion densities. This dipolarization is also accompanied by an higher frequency magnetic wave activity.

A strong magnetic activity is also present below $10 \mathrm{~Hz}$ between the ion cyclotron frequency (or also the electron bounce frequency) and the lower-hybrid frequency (Robert et al., 1984; Perraut et al., 1993; Shinohara et al., 1998; Sigsbee et al., 2001; Shiokawa et al., 2005). Low-frequency $\left(<\Omega_{i}\right)$ electromagnetic waves are also detected (Roux et al., 1991; Erickson et al., 2000). In the following sections, however, we focus on the higher frequency range namely the whistler frequency range $\left(\Omega_{i}<\omega<\left|\Omega_{e}\right|\right)$ which are detected before, during and after the local dipolarization.

\subsection{Polarization analysis}

Using the method developed by Samson and Olson (1980) we analyze the polarization characteristics of magnetic fluctuations recorded by the near-earth probes (THD and THE) during the burst period. Figure 4 shows again the magnetic field observations from FGM (panels a, b, c, and d) and displays the results of the wave polarization analysis between 02:20 and 02:32 UT. Panels (e) and (f) show the power spectral densities of the magnetic fluctuations obtained from 


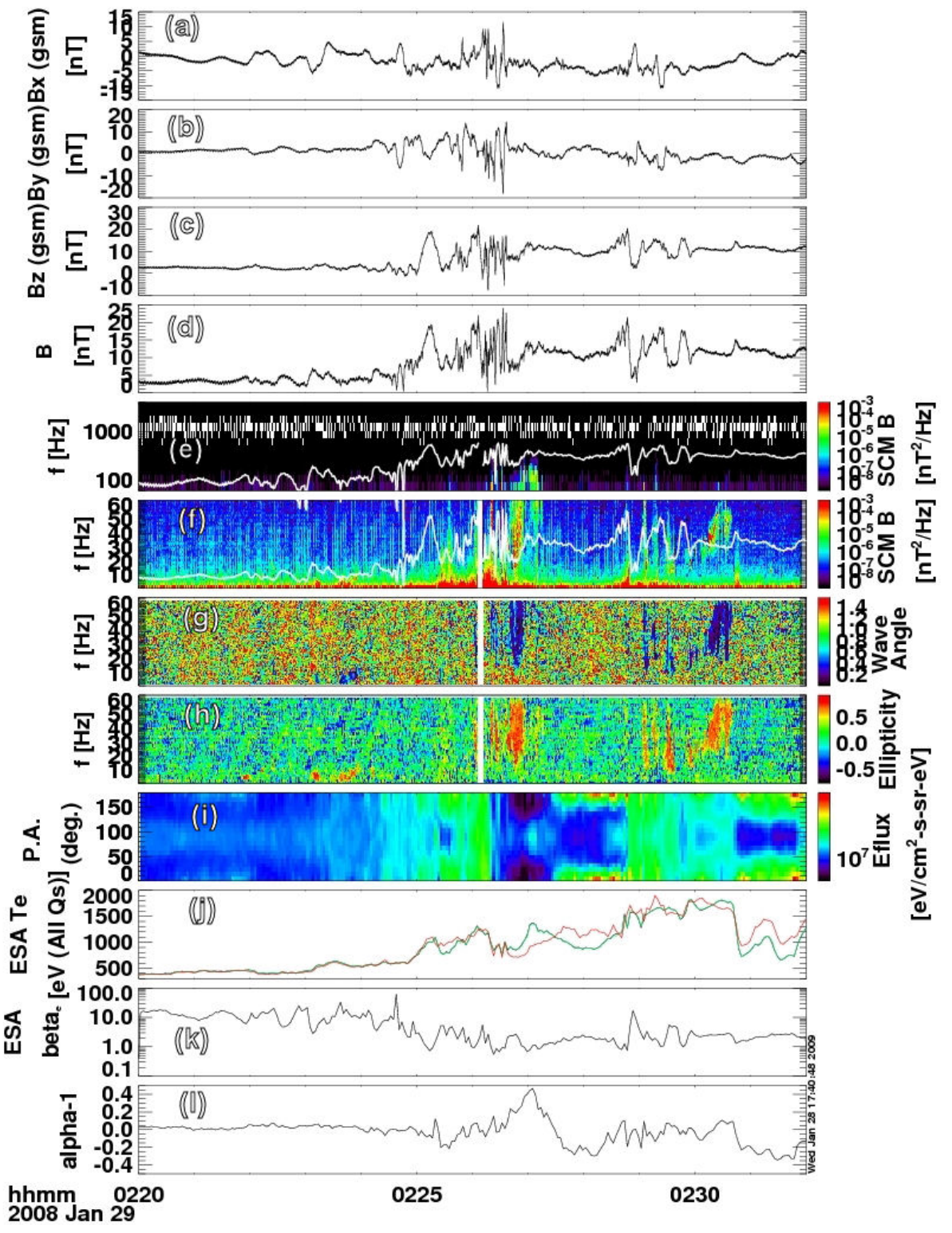

Fig. 4. Panels (a, b, c, d): three components and modulus of the magnetic field (128 S/s) in GSM coordinates, (e) $B$ power spectral densities from onboard FFT and (f) from SCM waveform capture (one spectrum every 1 s), White curve on panel (e) (resp. on panel f) correspond to $\left|\Omega_{e}\right| /(2 \pi)$ (resp. $0.1\left|\Omega_{e}\right| /(2 \pi)$ ). Note that the frequency scale is logarithmic on panel (e) and linear on panel (f). Panel (g) displays the angle between the wave vector and the background magnetic field (1s averaging), (h) ellipticity, (i) electron pitch-angle anisotropy spectrogram (ESA), (j) perpendicular (black and green lines) and parallel (red line) electron temperatures (ESA), (k) electron parallel $\beta$, and (l) $\alpha-1$ anisotropy parameter.

onboard FFT (Cully et al., 2008) up to $4 \mathrm{kHz}$ and from waveform capture between 1 and $64 \mathrm{~Hz}$. Spurious noise on the SCM waveforms has been reduced thanks to a noise reduction process (Le Contel et al., 2008). The waveform has been converted from the spacecraft frame (DSL) to a magnetic field aligned (MFA) frame. This MFA frame is computed using a $1 \mathrm{~s}$ averaged magnetic field. The $\mathrm{z}$-component corresponds to the magnetic field direction, y-direction is ob- tained from the cross product of the $z$ vector and the GSM $\mathrm{x}$-direction and the last one is the cross product of the first two. This waveform expressed in the MFA frame is used to compute the spectral matrix and the polarization characteristics (Samson and Olson, 1980). The time resolution of the spectra is $1 \mathrm{~s}$. Broad and narrow band emissions are detected in the whistler frequency range between 1 and $300 \mathrm{~Hz}$. These emissions correspond to a degree of polarization close 


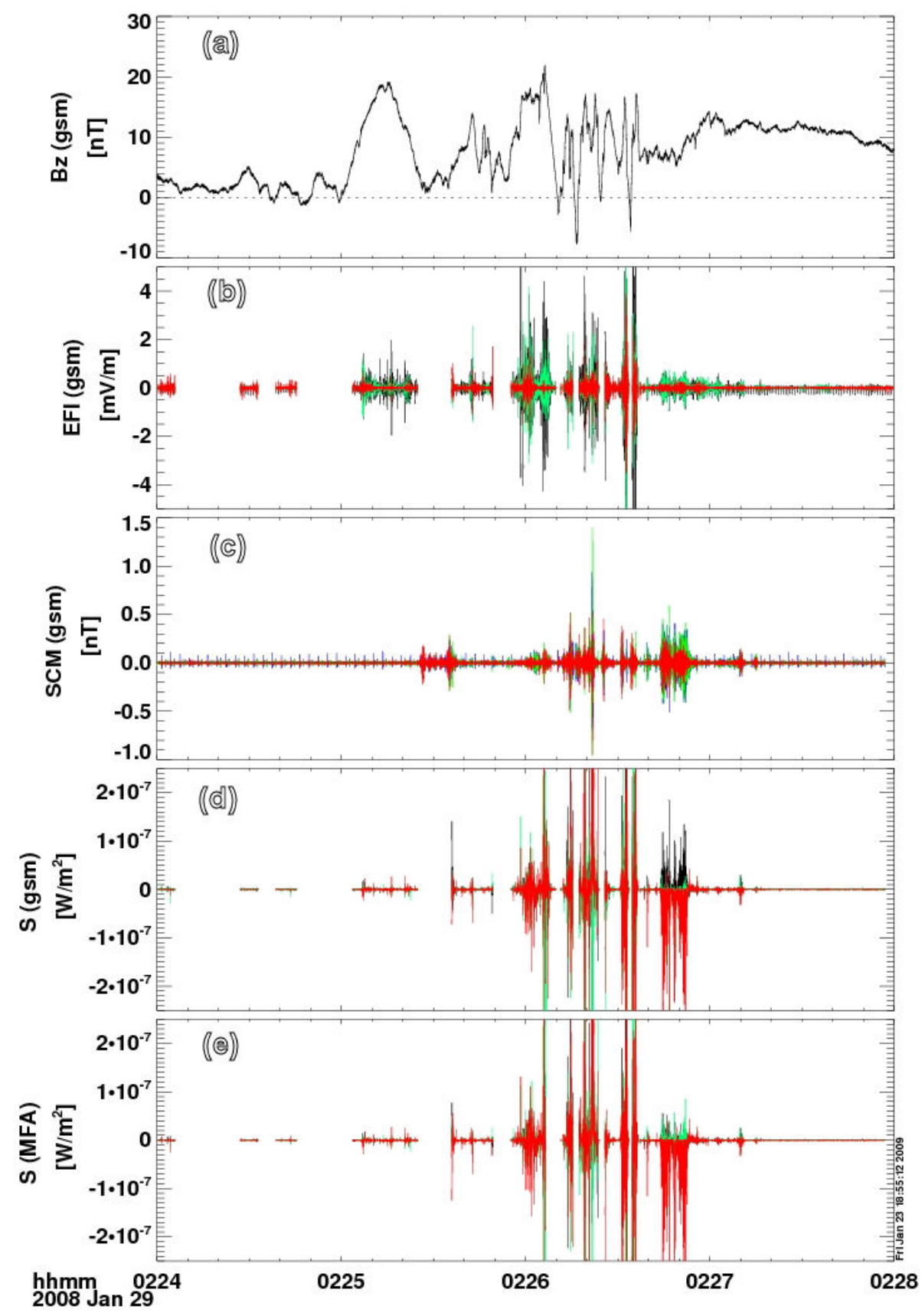

Fig. 5. Panels (a) $B_{z}$ component (128 S/s) in GSM coordinates, (b) EFI and (c) SCM waveforms (128 S/s) filtered below $10 \mathrm{~Hz}$ in GSM coordinates (x:black, y:green, z:red), Poynting vector in GSM (d) and MFA coordinates (e).

to 1 (not shown), a wave angle (the angle between the wave vector and the background magnetic field) close to 0 (blue in panel g) and an ellipticity close to 1 (red in panel h). Panels (i), (j), (k), and (l) will be described in Sect. 3. The ellipticity is equal to the ratio between the major and the minor axes of the ellipse of polarization. It is equal to +1 (resp. -1) for a wave which is right-hand (resp. left-hand) circularly polarized. Thus most of the emissions detected in the whistler frequency range are identified as right-hand circularly polarized waves propagating in a direction quasi-parallel to the ambient magnetic field. However waves detected below $20 \mathrm{~Hz}$ around 02:26 UT and just before 02:29 UT during large amplitude low-frequency magnetic fluctuations do not display any clear polarization signature.

Note that weak whistler emissions below $15 \mathrm{~Hz}$ are detected at the beginning of the period $(\simeq 02: 22,02: 23: 20$, and 


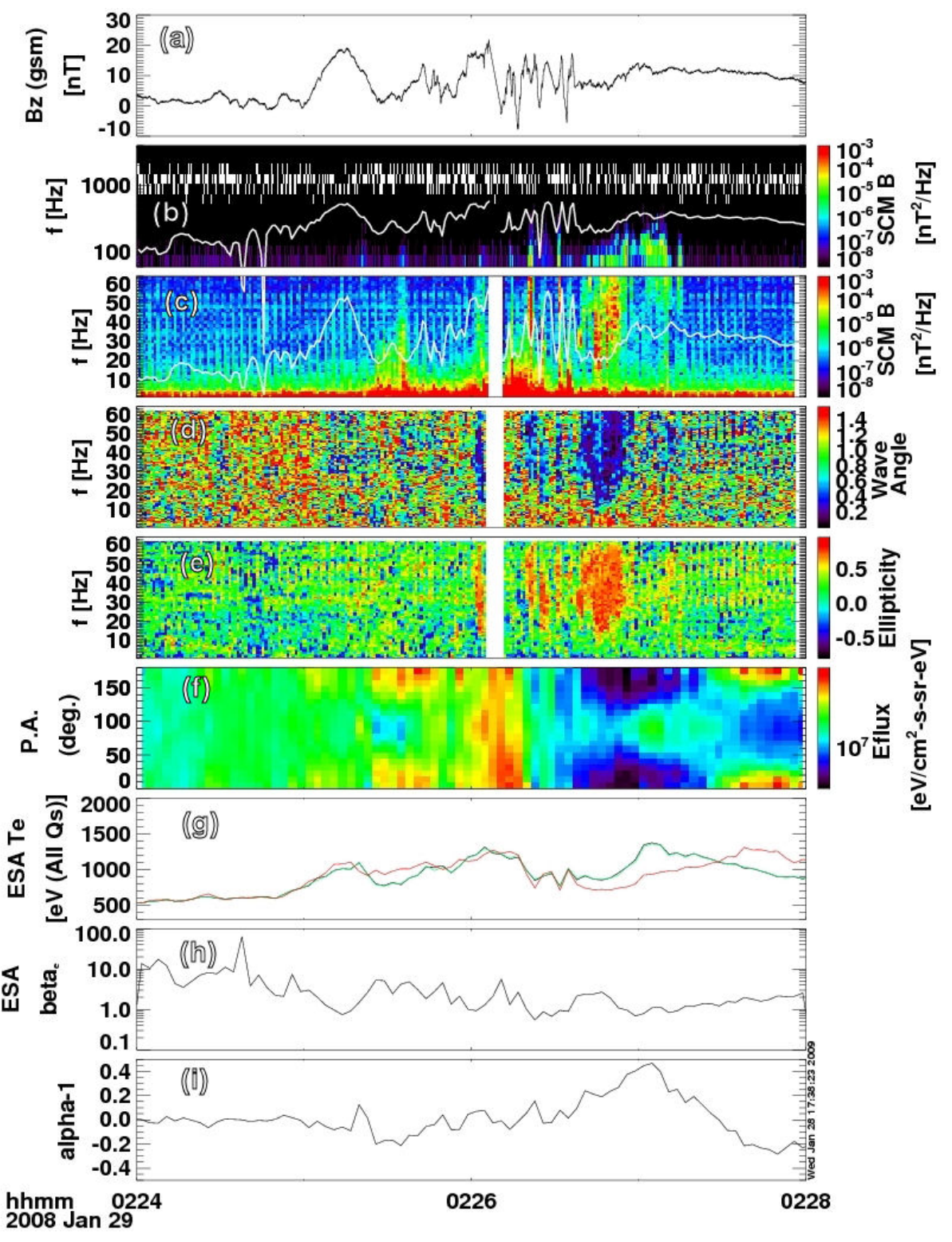

Fig. 6. Panel (a) displays the $B_{z}$ component ( $\left.128 \mathrm{~S} / \mathrm{s}\right)$ in GSM coordinates, then same legend as Fig. 4

02:23:40) before the local onset. The frequency band of the emissions is relatively narrow and increases as the modulus of $B$ increases. These emissions are recorded when the $B_{x}$ component of the magnetic field is small indicating that their source is close to the magnetic equator. Broad band emissions without frequency dispersion are detected at higher frequencies (up to $300 \mathrm{~Hz}$ ) during and after the dipolarization suggesting again that the sources are very close to the probe. Finally the second low-frequency wave train is also associated with broad band whistler emissions without dispersion while the last whistler emissions between 02:30:10 and 02:30:30 UT present a small dispersion; emissions around $30 \mathrm{~Hz}$ being detected before emissions around $50 \mathrm{~Hz}$.
This wave polarization analysis provides the direction of propagation with an ambiguity of $180^{\circ}$. In the next section we use the electric field measurement to compute the Poynting vector associated with the whistler emissions in order to distinguish between parallel and anti-parallel wave propagations.

\subsection{Poynting vector calculations}

In this section we compute the Poynting vector of the emissions in the whistler frequency range. We use two components of the electric field provided by the spin plane double probes (Bonnell et al., 2008). The third component can be 
computed from the assumption $\mathbf{E} \cdot \mathbf{B}_{0}=0$ (as long as the angle between the spin axis and the magnetic field is smaller than a maximum value here fixed at $80^{\circ}$ ). This assumption is valid for such quasi-parallel whistler waves. Electric field as well as magnetic field waveforms are filtered to remove waves below $10 \mathrm{~Hz}$ in order to focus on intense wave emissions in the whistler frequency range. Figure 5 shows $B_{z}$ from FGM (panel a), the electric (panel b) and magnetic (panel c) field waveforms filtered between $10 \mathrm{~Hz}$ and $64 \mathrm{~Hz}$ in GSM coordinates. Poynting vector calculations in GSM and MFA coordinates are plotted in panels (d) and (e). Large amplitude electric and magnetic field fluctuations are recorded around 02:26 UT but their polarization characteristics are not well determined probably due to the fast variations of the background magnetic field (see Fig. 4). The whistler waves detected between 02:26:40 and 02:27:00 UT with a clear polarization seen on Fig. 4 correspond to an electric (resp. magnetic) field amplitude of about $0.5 \mathrm{mV} / \mathrm{m}$ (resp. $0.5 \mathrm{nT}$ ) leading to a rough estimate of the phase velocity of $1000 \mathrm{~km} / \mathrm{s}$. The corresponding Poynting vector calculations $\mathbf{S}=\delta \mathbf{E} \times \delta \mathbf{B} / \mu_{0}$ (where $\mu_{0}$ is the magnetic permeability of the vacuum) indicate that the wave energy moves mostly along the $x$ and $-z$ GSM directions (panel d), anti-parallel $\left(S_{\|} \simeq-2 \times 10^{-7} \mathrm{~W} / \mathrm{m}^{2}\right)$ to the magnetic field (panel e). THD being located slightly southward of the magnetic equator of the current sheet $\left(B_{x} \simeq-2.5 \mathrm{nT}\right.$ and $\left.B_{z} \simeq 8 \mathrm{nT}\right)$ it means that the whistler mode waves are propagating towards the earth. The same signatures are observed on THE data just before local dipolarization between 02:25:00 and 02:25:30 UT and also between 02:26:10 and 02:26:45 UT (not shown). The parallel component of the Poynting vector is positive and reaches $10^{-7} \mathrm{~W} / \mathrm{m}^{2}$; THE being located northward of the magnetic equator it corresponds again to a whistler mode propagating earthward. Thus the Poynting vector calculations indicate that the whistler mode waves detected by the near-earth probes (THD and THE) of the THEMIS mission propagates along the magnetic field towards the earth. In the next section we consider the electron temperature anisotropy as a possible mechanism to generate these broad and narrow band quasi-parallel whistler waves.

\section{Discussion}

In a hot plasma the temperature anisotropy $\alpha \equiv T_{\perp e} / T_{\| e}>1$ is the source of whistler anisotropy instability (Kennel and Petschek, 1966; Roux and Solomon, 1971; Gary, 1993; Gary and Wang, 1996). A necessary condition for the growth of the WAI was given by Kennel and Petschek (1966) as:

$\alpha-1>\frac{1}{\left|\Omega_{e}\right| / \omega_{r}-1}$.

This instability grows at real frequencies between the proton and electron cyclotron frequencies. Its maximum growth rate is obtained for strictly parallel or anti-parallel propagation.
For a purely parallel or anti-parallel propagation the associated electromagnetic fluctuations are transverse, right-hand circularly polarized, and have their phase velocity and Poynting vector directions aligned. All these properties are satisfied by most of the electromagnetic fluctuations detected in the whistler frequency range $\left(\Omega_{i}<\omega<\left|\Omega_{e}\right|\right)$ by the near-earth THEMIS satellites (THD and THE) before, during and after the local dipolarization (Fig. 4). Panel (j) on Fig. 4 shows that whistler emissions are detected when the perpendicular temperatures (black and green lines) are larger than the parallel one (red line). As predicted by the relation (1) the upper frequency of the whistler emission (panels e and f) increases with the anisotropy (panel 1).Furthermore as pointed out by Gary and Wang (1996) theory predicts that the threshold electron anisotropy varies inversely with $\beta_{\| e}$. Using two-dimensional particle-in-cell simulations Gary and Wang (1996) showed that an electron anisotropy upper bound is imposed by wave-particle scattering which takes the general form:

$\frac{T_{\perp e}}{T_{\| e}}-1=\frac{S_{e}}{\beta_{\| e}^{\alpha_{e}}}$,

where $S_{e}$ and $\alpha_{e}$ are fitting parameters and depend on the choice of the ratio $\gamma_{m} /\left|\Omega_{e}\right|$ ( $\gamma_{m}$ being the maximum growth rate). Note that the $\alpha_{e}$ fitting parameter used by Gary and Wang (1996) is not to be confused with $\alpha$ the electron temperature anisotropy. The parameter $\beta_{\| e}$ is plotted on panel (k) in Fig. 4. From 02:20 to $02: 25$ UT $\beta_{\| e} \simeq 10$ corresponding to the period when the satellite is very close to the magnetic equator. Such a high $\beta_{\| e}$ value allows the growth of the WAI, even with a small anisotropy. Indeed narrow band whistler emissions detected below $20 \mathrm{~Hz}$ before $02: 25$ UT correspond to $\alpha \simeq 1.05$. After $02: 25 \mathrm{UT} \beta_{\| e}$ decreases to values around 1 due to the increase of $B_{z}$ component of the magnetic field and to the decrease of the pressure associated with the local dipolarization. During this period whistler emissions are associated with larger anisotropies. Thus as shown theoretically by Gary and Wang (1996) fast wave-particle scattering by enhanced whistler fluctuations for high $\beta_{\| e}$ limits the electron anisotropy $\alpha$ while low $\beta_{\| e}$ values let larger anisotropies to develop. Note also that the largest frequency is reached around 02:27 UT corresponding to the smallest $\beta_{e}$ value and to the largest $\alpha$ value.

In order to compare observations with the theory of the whistler mode instability, we solve the dispersion relation for linear waves using the WHAMP code (Rönnmark, 1982). Two different plasma models are considered in order to take into account the evolution of the near-earth tail before (from 02:20 to 02:25 UT) and after (from 02:25 to 02:32 UT) the local dipolarization. As described in Sect. 2.2 THD is located close to the magnetic equator from 02:20 to $02: 25$ UT. Particle densities are quite large $\left(\simeq 0.75 \mathrm{p} / \mathrm{cm}^{3}\right)$ whereas the electron (resp. ion) temperature is about $400 \mathrm{eV}$ (resp. $5 \mathrm{keV}$ ). We choose $B_{0}=4 \mathrm{nT}$ as an average value of the background magnetic field during this period. Taking 


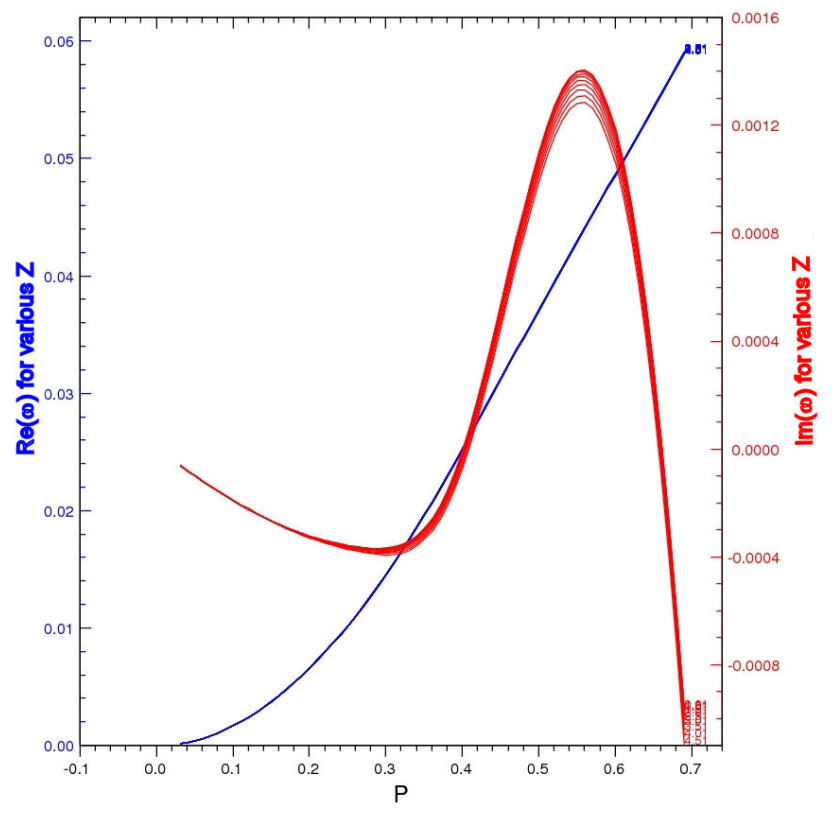

Fig. 7. Results for model 1: Real frequency $\operatorname{Re}(\omega) / \Omega_{e}$ (blue line) and damping/growth rates $\operatorname{Im}(\omega) / \Omega_{e}$ (red line) of the whistler anisotropy instability as function of the modulus of the wave number $P=k \rho_{e}$ and for different wave angle (Z) between 0 and $5^{\circ}$.

into account the chosen electron temperature, this gives a large $\beta_{\|_{e}} \simeq 7.5$ (see Table 1 for a summary of the parameters). Figure 7 displays the results from WHAMP, for the first plasma model; it shows that for a small electron anisotropy $(\alpha=1.06)$ we obtain a maximum growth rate $\gamma_{m} \simeq 0.0014\left|\Omega_{e}\right|$ for a real frequency $\omega_{r} \simeq 0.044\left|\Omega_{e}\right|$. The bandwidth (from $\simeq 0.025$ to $\simeq 0.055\left|\Omega_{e}\right|$ or from $\simeq 2.8$ to $\simeq 6.2 \mathrm{~Hz}$ with $\left.\left|\Omega_{e}\right| /(2 \pi) \simeq 113 \mathrm{~Hz}\right)$ corresponding to $\gamma \geq 0$ is consistent with the observed bandwidth on Fig. 4. Figure 8 displays WHAMP results for the second plasma model corresponding to the period after the local dipolarization (from 02:25 to 02:32 UT), when THD is farther from the equator. In particular we have taken the plasma parameters corresponding to the period during which the Poynting vector is clearly directed earthward (between 02:26:40 and 02:27 UT). In order to better illustrate the relation between the key parameters, $\alpha$ and $\beta_{\| e}$, we have expanded Fig. 4 between 02:24 and 02:28 UT on Fig. 6. Particle densities are smaller $\left(\simeq 0.5 \mathrm{p} / \mathrm{cm}^{3}\right)$, the electron parallel temperature is larger $(700 \mathrm{eV})$ while the ion temperature is taken unchanged $(5 \mathrm{keV})$ in order to focus on the effect of the electron temperature. However the local dipolarization process increases the ion temperature too (up to $6 \mathrm{keV}$ ). Due to the very large $\beta_{i} \simeq 100$ value the results cannot be assumed to be independent of the $T_{e} / T_{i}$ ratio but we have checked that it has little effect on the results. A larger average value of the background magnetic field $B_{0}=8 \mathrm{nT}$ is taken during this period, in order to account for the effect

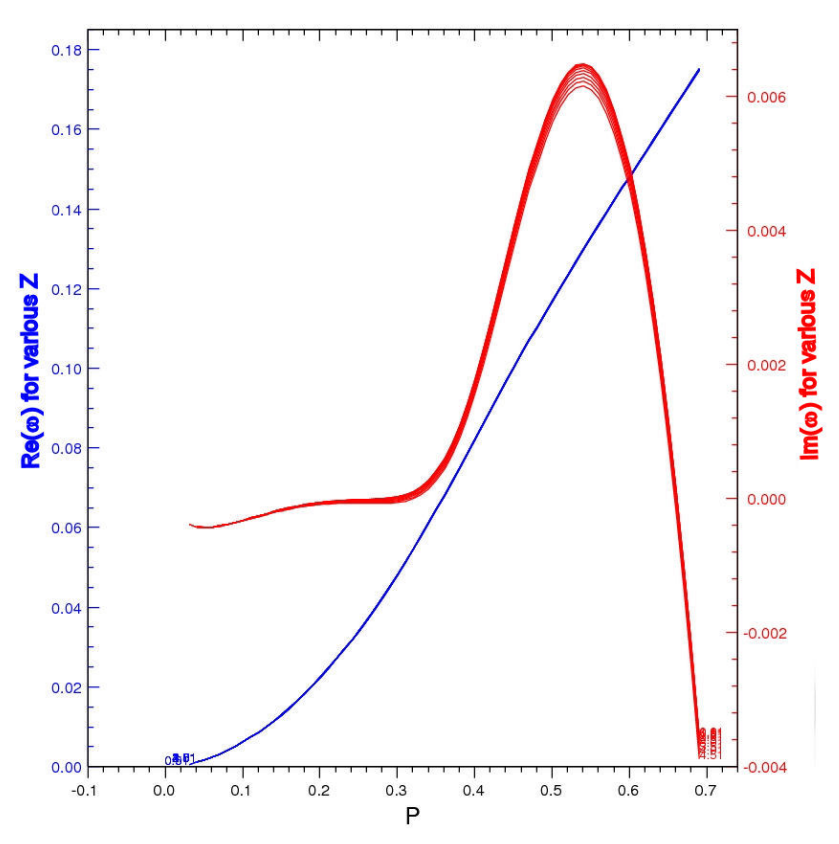

Fig. 8. Results for model 2. Same legend as Fig. 7

Table 1. Plasma model 1 corresponding to the period between 02:20 and 02:25 UT with $B_{0}=4 \mathrm{nT}$.

\begin{tabular}{ccccc}
\hline Species & Density $\left(\mathrm{cm}^{-3}\right)$ & $T_{\|}(\mathrm{eV})$ & $\alpha$ & $\beta_{\|}$ \\
\hline $\mathrm{H}+$ & 0.75 & 5000 & 1 & 94 \\
$\mathrm{e}-$ & 0.75 & 400 & 1.06 & 7.5 \\
\hline
\end{tabular}

of the dipolarization (see Table 2). These parameters lead to a smaller $\beta_{\| e} \simeq 2.2$. We obtain now $\gamma_{m} \simeq 0.006\left|\Omega_{e}\right|$ for $\omega_{r} \simeq 0.13\left|\Omega_{e}\right|$. This value is consistent with the Fig. 1 of Gary and Wang (1996) showing the electron temperature anisotropy, as a function of $\beta_{\| e}$ if we extrapolate the curve for $\gamma_{m} \simeq 0.006\left|\Omega_{e}\right|$. The parallel phase velocity at the maximum growth rate is about $0.24 v_{e} \simeq 1880 \mathrm{~km} / \mathrm{s}$; this is of the order of magnitude as the estimate obtained from the observations presented in Sect. 2.4. The bandwidth corresponding to $\gamma>0$ is now from $\simeq 0.07$ to $0.17\left|\Omega_{e}\right|$ which is equivalent to a bandwidth from $\simeq 16$ to $38 \mathrm{~Hz}$ not fully consistent with the broad band signature of the whistler waves detected up to $64 \mathrm{~Hz}\left(\simeq 0.3\left|\Omega_{e}\right|\right)$ during this period (see Fig. 6). Furthermore whistler mode waves are expected to propagate in both the parallel and the anti-parallel directions.

In order to go further in the comparaison between the present observations and the linear theory, we assume, as a first step, that the electron anisotropy is constant along the magnetic field line around the location of THD. Whistler mode waves emitted farther from the equator than the location of THD (but along the same field line) will have higher frequencies; around a frequency that corresponds 
Table 2. Plasma model 2 corresponding to the period between $02: 25$ and $02: 32 \mathrm{UT}$ with $B_{0}=8 \mathrm{nT}$.

\begin{tabular}{ccccc}
\hline Species & Density $\left(\mathrm{cm}^{-3}\right)$ & $T_{\|}(\mathrm{eV})$ & $\alpha$ & $\beta_{\|}$ \\
\hline $\mathrm{H}+$ & 0.5 & 5000 & 1 & 16 \\
$\mathrm{e}-$ & 0.5 & 700 & 1.2 & 2.2 \\
\hline
\end{tabular}

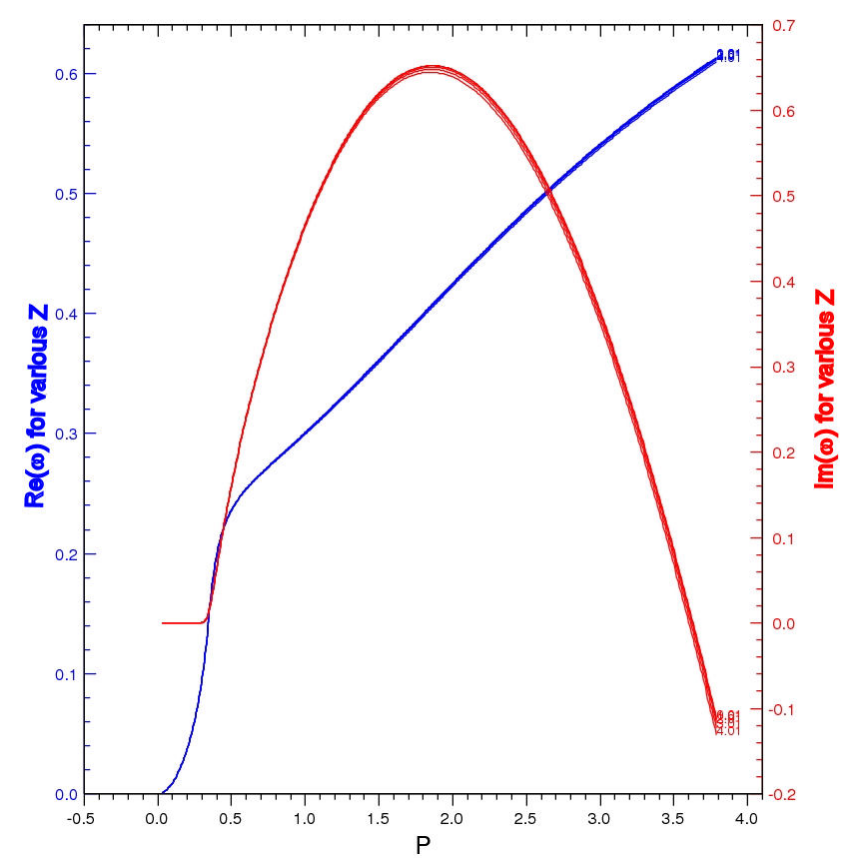

Fig. 9. Results for model 3. Same legend as Fig. 7

typically to $0.1\left|\Omega_{e}\right|$, for $B_{0}=16 \mathrm{nT}$. Note, however, that their growth rates will be smaller due to the decrease of $\beta_{\|_{e}}$. As they reach the location of THD $\left(B_{0}=8 \mathrm{nT}\right)$ these frequencies will correspond to $\simeq 0.1\left|\Omega_{e}(16 \mathrm{nT})\right| \simeq 0.2\left|\Omega_{e}(8 \mathrm{nT})\right|$ and will be strongly damped (see Fig. 8). On the other hand, whistler mode waves emitted closer to the magnetic equator at frequencies $\simeq 0.1\left|\Omega_{e}(4 \mathrm{nT})\right| \simeq 0.05\left|\Omega_{e}(8 \mathrm{nT})\right|$ will correspond to slightly positive growth rates (see Fig. 8). Thus at the location of THD whistler mode waves propagating away from the magnetic equator will be dominant whereas whistler mode waves propagating toward the equator will be damped. This simple description accounts for the whistler mode wave propagation towards the Earth, but it fails to account for the high frequency part of the whistler emissions $\left(\simeq 0.3\left|\Omega_{e}(8 \mathrm{nT})\right|\right)$.

Now, let us consider that the electron temperature anisotropy is no longer constant along the field line. Following an approach based on the Liouville's theorem developed by Chiu and Schulz (1978), we assume the system to be time-independent at the time scale of the whistler mode wave generation and the particle dynamics to be adiabatic
Table 3. Plasma model 3 corresponding to the period between 02:25 and 02:32 UT closer to the equator with $B_{0}=4 \mathrm{nT}$.

\begin{tabular}{ccccc}
\hline Species & Density $\left(\mathrm{cm}^{-3}\right)$ & $T_{\|}(\mathrm{eV})$ & $\alpha$ & $\beta_{\|}$ \\
\hline $\mathrm{H}+$ & 0.5 & 5000 & 1 & 64 \\
$\mathrm{e}-$ & 0.5 & 700 & 2.5 & 8.8 \\
\hline
\end{tabular}

(the scale of the magnetic field gradient is assumed to be larger than the particle Larmor radius). Then the electron distribution function must depend only on the constants of the motion, namely the kinetic energy $\left(W=m\left(v_{\|, s}^{2}+v_{\perp, s}^{2}\right) / 2\right)$ and the magnetic moment $\left(\mu=m v_{\perp, s}^{2} /(2 B(s))\right)$ where $s$ is the coordinate that measures the arc length of the field line from the equator. From the Liouville's theorem we can write that $f\left(v_{\|, e q}, v_{\perp, e q}\right)=f\left(v_{\|, s}, v_{\perp, s}\right)$ where the subscript $e q$ denotes the equatorial location $(s=0)$, if the points $\left(v_{\|, e q}, v_{\perp, e q}, e q\right)$ and $\left(v_{\|, s}, v_{\perp, s}, s\right)$ are connected by a dynamical trajectory in phase space. Assuming that the equatorial distribution function writes as $f\left(v_{\|, e q}, v_{\perp, e q}\right) \propto \exp \left(m\left(v_{\|, e q}^{2} / T_{\|, e q}+v_{\perp, e q}^{2} / T_{\perp, e q}\right) / 2\right) \quad$ we get:

$$
\begin{aligned}
T_{\|}(s) & =c s t, \\
\alpha(s) & =\frac{\frac{B(s)}{B_{e q}} \alpha_{e q}}{\frac{B(s)}{B_{e q}} \alpha_{e q}-\left(\alpha_{e q}-1\right)} .
\end{aligned}
$$

This relation has been also demonstrated using a fluid approach by Passot et al. (2006). Note that to avoid treating the parallel electric field ( $W$ does not contain the term of the electrostatic energy) we have implicitly assumed that electrons and ions have the same anisotropy (Persson, 1966). From the relation (4) and the measured electron anisotropy of 1.2 at $B_{0}=8 \mathrm{nT}$, we get that the electron temperature anisotropy at the equator, where $B_{0}=4 \mathrm{nT}$, is 1.5 instead of 1.2. For this plasma model $(\alpha=1.5$ and $B_{0}=4 \mathrm{nT}$ ), WHAMP results (not shown) indicate that the bandwidth corresponding to $\gamma>0$ is now from $\simeq 0.05$ to $0.34\left|\Omega_{e}(4 \mathrm{nT})\right|$. This bandwidth is still not fully consistent with the observations which correspond to an upper frequency $\simeq 0.6\left|\Omega_{e}(4 \mathrm{nT})\right| \simeq 0.3\left|\Omega_{e}(8 \mathrm{nT})\right|$. From relation (1), we know that $\alpha \simeq 2.5$ (at the equator) is needed to get $\omega \simeq 0.6\left|\Omega_{e}\right|$ and Fig. 9 displays the WHAMP results for the corresponding plasma model 3 (see Table 3). The bandwidth with $\gamma>0$ (up to $0.66\left|\Omega_{e}\right|$ ) is now from $\simeq 0.14$ to $0.6\left|\Omega_{e}(4 \mathrm{nT})\right|$ or $\simeq 0.07$ to $0.3\left|\Omega_{e}(8 \mathrm{nT})\right|$ which is consistent with the observations. It is a realistic value for the nearearth tail region during substorms (Kremser et al., 1986), and it is smaller than the anisotropies $\alpha \simeq 3-7$ used in numerical simulations (Devine et al., 1995; Gary and Wang, 1996; Katoh and Omura, 2006; Sydora et al., 2007). Using relation (4) such a large electron temperature anisotropy would correspond to $\alpha=1.42$ at the location of THD $\left(B_{0}=8 \mathrm{nT}\right)$ and 
to $\alpha=1.18$ at $B_{0}=16 \mathrm{nT}$. WHAMP results (not shown) for $\alpha=1.42$ with $B_{0}=8 \mathrm{nT}$ and for $\alpha=1.18$ with $B_{0}=16 \mathrm{nT}$ indicate that the whistler mode waves emitted at the equator with $\omega \simeq 0.6\left|\Omega_{e}(4 \mathrm{nT})\right|$ still have a slightly positive growth rate at $B_{0}=8 \mathrm{nT}$ (with $\alpha=1.42$ ) whereas the whistler mode waves emitted at $B_{0}=16 \mathrm{nT}$ (with $\alpha=1.18$ ) for $\omega \simeq 0.15\left|\Omega_{e}(16 \mathrm{nT})\right|$ have a very small positive growth rate not larger than $2 \times 10^{-5}\left|\Omega_{e}(16 \mathrm{nT})\right|$. Therefore the results from the linear theory with $\alpha \simeq 2.5$ at the equator (corresponding to 1.42 at the location of THD from the relation 4) are consistent with the bandwidth and the earthward propagation of the whistler mode waves observed by THD. The discrepancy between the measured electron temperature anisotropy (1.2) and the estimate from Liouville's theorem (1.42) can be due to the assumption of stationarity which is not adapted during substorm periods. Indeed panel (i) of Fig. 6 shows that the electron temperature anisotropy increases from 1.2 to 1.4 between 02:26:40 and 02:27:00 UT. Also, the assumption of the same temperature anisotropies for ions and electrons (which implies that there is no parallel electric field) may be not appropriate. Finally, recent self-consistent numerical simulations with a nonuniform magnetic field (dipole) showed that whistler mode waves are generated from the equator through the WAI and that nonlinear effects (interaction of the whistler mode waves with the bouncing electrons) can produce an increase of the frequency in the region close to the equator (Katoh and Omura, 2006, 2007; Omura et al., 2008). The authors concluded that the nonuniformity of the magnetic field near the equator plays a key role in the amplification process of whistler mode wave emissions formed at the equator. While these studies are initialized with parameters corresponding to the Chorus emission region $\left(|X| \simeq 4 R_{E}\right)$, one can expect that these effects are also present in the near-earth tail region. Indeed here the ratio between the wave amplitude and the background magnetic field is about $6.25 \times 10^{-2}$ (Fig. 5) whereas this ratio is $\simeq 10^{-4}$ in the region of the Chorus emissions.

Stenberg et al. (2005) suggested that the direction of whistler mode wave propagation was related to the asymmetry of the electron distribution function with regard to $v_{\|}=0$. In their case, the electron space density being smaller in the direction parallel to the magnetic field than anti-parallel and perpendicular, the whistler mode waves should propagate in the direction anti-parallel as observed close to the magnetopause. However, we have not found any evidences for such an asymmetry in THEMIS particle data.

We verify a posteriori from these WHAMP calculations that the assumption $\mathbf{E} \cdot \mathbf{B}_{0}=0$ used to compute the Poynting vector is satisfied by these whistler waves generated by electron temperature anisotropy. All models except the model 3 give small parallel electric fields from $5 \times 10^{-4}$ to $0.1 E_{\perp}$, ( $E_{\perp}$ being the perpendicular component of the wave electric field) for the propagation angles under consideration (from 0.01 to $5^{\circ}$ ). The parallel electric field obtained from the model 3 can reach $0.4 E_{\perp}$ for a propagation angle of $5^{\circ}$.
Thus the results from these plasma models seem to confirm that the whistler mode wave emissions detected by THD are consistent with the dependence of the threshold of the linear WAI on the $\beta_{\|_{e}}$ parameter. A smaller $\beta_{\| e}$ requires a larger electron anisotropy $\alpha$. The bandwidth of the whistler emissions is related to the anisotropy, being broader for a larger anisotropy. This behaviour is expected as the upper limit given by Eq. (1) increases with $\alpha$. A change of $\beta_{\| e}$ from 7.5 to 2.2 for a constant anisotropy $(\alpha=1.2)$ does not strongly modify the bandwidth.

These whistler emissions detected by the near-earth probes are consistent with the predictions of the linear theory of WAI if we assume that a large electron temperature anisotropy ( $\alpha \simeq 2.5$ ) exists near the equator. However, the non uniformity of the magnetic field and the associated non linear effects should be taken into account in order to fully describe the amplification and the frequency range of the whistler mode wave emissions. Indeed, the nature of their generation mechanism seems to be very similar to that of the chorus emissions observed also near the equator of regions closer to the earth and related to electron anisotropy though loss cone instability can be also invoked (Parrot et al., 2003; Santolík et al., 2004; Li et al., 2008).Finally, recent developments by Sydora et al. (2007) showed that the non linear effects can also be present with a uniform magnetic field leading to coherent structures and modifying the frequency range of the whistler mode wave emissions.

The role of whistler emissions in the mid-tail during substorm process is still unclear. In the Hall reconnection model, the electron temperature anisotropy could increase the growth rate of the tearing instability and would allow smaller scale reconnection. This anisotropy could be produced by the nonlinear development of LHDI (Daughton et al., 2004). WAI having large growth rate and low threshold especially at large $\beta_{\| e}$ values, the WAI threshold anisotropy should be considered as an upper limit of the electron temperature anisotropy (Gary and Karimabadi, 2006). On the other hand, the electron acceleration by the whistler wave, invoked in the Hall reconnection model, can only happen for large frequencies, typically when $\omega>\left|\Omega_{e}\right| / 2$ (Kennel and Petschek, 1966). Otherwise only isotropization via pitchangle scattering occurs with little acceleration. For the four events considered here the mid-tail probes were located far from the magnetic equator and were never in burst mode, preventing detailed studies in the highest frequency part of the whistler frequency range in these regions. Yet, observations from filter bank data (not shown) indicate that there was no magnetic activity in the whistler frequency range in the mid-tail when the probes are far from the equator. Angelopoulos et al. (2008b) has recently published a case study for which mid-tail probes are located closer to the magnetic equator and particle burst mode are triggered too. For this event mid-tail THEMIS probes (THB and THC) observations show the same quasi-parallel whistler wave emissions, associated with current sheet oscillations, as those observed by 
Cluster (Le Contel et al., 2006; Wei et al., 2007) or by the THEMIS near-earth probes in the present paper. However the relation between these emissions and the electron temperature anisotropy is not clear as the current sheet oscillates very quickly; the relation with small scale current sheets is difficult to investigate from single spacecraft measurements. The detailed study of the quasi-parallel whistler wave emission observed by THEMIS in the mid-tail region will be addressed in a future paper.

As in the case of Cluster mid-tail observations described by Le Contel et al. (2006); Wei et al. (2007), we observe quasi-parallel whistler emissions before, during and after the local dipolarization occuring in the near-earth region. The source of these emissions is related to the electron temperature anisotropy $(\alpha>1)$. The origin of this anisotropy could be the nonlinear development of LHDI as electromagnetic fluctuations are also detected in this frequency range. Although, as mentioned early by Kennel and Petschek (1966), any mechanism that increases the particle pitch-angle such as magnetic field compression can make the electron distribution unstable to the whistler mode. To our knowledge the dependence of current driven instabilities on the electron temperature anisotropy has not been studied. Notably it would be interesting to know whether the instability conditions of the kink instability are modified. The role of whistler mode waves in the near-earth region could also be to control the threshold of a lower frequency instability which would be sensitive to the electron temperature anisotropy. Yet from the energetic point of view, Pellat's conclusions (Pellat et al., 1991) about the absence of tearing mode growth should remain valid whatever the low-frequency $\left(\omega<\omega_{b, e}\right)$ instability considered. Electron pitch-angle scattering by whistler waves will not change the number of electrons in the flux tube; therefore this process will not modify the energy principle. Indeed, in the tail, the loss cone is extremely small, hence the minimum precipitation lifetime is so long that particle losses from a flux tube are negligible.

On the other hand, as already mentioned, Cluster observations in the mid-tail and close to the magnetopause suggest that such whistler mode emissions can be associated with small scale $\left(<\rho_{i}\right.$ or $\left.<c / \omega_{p i}\right)$ current sheets. Single satellite measurements do not allow to disentangle temporal and spatial variations. Yet the fast variations of the low-frequency magnetic field associated with the local dipolarization do not rule out the possible existence of such small scale current sheets embedded in the near-earth tail. In that case the homogenous linear theory would no longer be applicable. The development of a theory, valid for a nonuniform magnetic field, is out of the scope of this paper but it is worthwile mentioning that Bulanov et al. (1992) has already studied this problem using the electron magnetohydrodynamics approach. More recently Attico et al. (2002) used a full kinetic approach for modelling laboratory experiments. These studies showed that small-scale $\left(c / \omega_{p e}<l<c / \omega_{p i}\right)$ magnetic reconnection can occur in the whistler frequency range or in other words that small current sheets $\left(c / \omega_{p e}<H<c / \omega_{p i}\right)$ are unstable to tearing mode with $k H \simeq 1$ in the whistler frequency range ( $k$ being the wave vector). Therefore at this stage one cannot rule out the possibility of small scale tearing mode developing in the whistler mode range in the nearearth tail region. However, it would be only one element of the chain of processes as a larger scale instability is needed in order to produce a dipolarization at the larger scale. Finally whistler mode waves could be only a by product of the current sheet oscillations without any fundamental role in the substorm process. All these questions have to be further addressed in the future.

\section{Conclusions}

We have presented data from four near-earth dipolarization events captured by the THEMIS probes on 29 January 2008 between 02:00 and 03:50 UT. We have only described in detail the first event corresponding to a pseudo-substorm event but most of the results are valid for the other events as well as for already published substorm events analyzed by the THEMIS team (Angelopoulos et al., 2008b; Lui et al., 2008; Runov et al., 2008). We show from a polarization analysis that quasi-parallel whistler mode waves are detected before, during and after the local dipolarization by the near-earth probes (THD and THE). We observe locally an electron temperature anisotropy $\left(T_{\perp e}>T_{\| e}\right)$. The measured anisotropy is sufficient to drive the whistler mode unstable. In agreement with the linear theory, we also found that the observed electron temperature anisotropy associated with whistler mode waves varies inversely with the electron parallel $\beta_{\| e}$. Narrow band emissions are recorded for a small electron temperature anisotropy (and hence a large $\beta_{\| e}$ ) whereas broad band emissions correspond to large anisotropy values (and smaller $\beta_{\| e}$ ). Poynting vector calculations demonstrate that the energy in the whistler mode leaves the current sheet and propagates along the background magnetic field, towards the Earth. A simple time-independent description based on Liouville's theorem shows that the electron temperature anisotropy decreases with the distance along the field line from the equator. Therefore whistler mode waves will be hardly generated between the earth and the spacecraft due to small anisotropies. Conversely whistler mode waves generated near the magnetic equator, where the anisotropy is maximum, have very large growth rates and propagate towards the Earth. This asymmetry explains why the Poynting vector is directed earthward, while the local growth rate predicts amplification in both directions. Yet, the required anisotropy is larger than the one measured by ESA suggesting that timedependent effects could be important. On the other hand this discrepancy of about $20 \%$ can be due to the upper limit to the ESA's energy range which can be a source of error in the moment calculation as soon as energetic particle fluxes are not negligible (McFadden et al., 2008). Furthermore 
recent self-consistent numerical studies about chorus emissions showed that the nonuniformity of the magnetic field near the equator plays a key role in the amplification process (Katoh and Omura, 2007; Omura et al., 2008) of whistler mode wave emissions formed at the equator and that nonlinear effects can increase the frequency of the emissions. As a last comment: Cluster observations in the mid-tail and close to the magnetopause indicate that whistler emissions can be related to small scale current sheets. While present THEMIS observations indicate that the whistler mode wave emissions during relatively steady magnetic field (02:26:4002:27:00 UT) are consistent with the linear theory of WAI, some of the whistler emissions during very fast magnetic field variations (02:26:00-02:26:40 UT) could be associated with very thin current sheets. Therefore the whistler mode waves observed in the mid-tail as well as in the near-earth tail during substorms cannot be interpreted as the unambiguous signature of the Hall reconnection process. Thus the role of these whistler emissions in the dipolarization process or more generally in the substorm process still deserves future studies.

Acknowledgements. The French involvment on THEMIS is supported by CNES and CNRS-INSU. Work in the US was supported by NASA contract NAS5-02099. The work of KHG and UA at the Technical University of Braunschweig was financially supported by the German Ministerium für Wirtschaft und Technologie and the German Zentrum für Luft- und Raumfahrt under grant 50QP0402. The CARISMA array is operated and deployed by the University of Alberta, funded by the Canadian Space Agency.

Topical Editor R. Nakamura thanks R. Lysak and another anonymous referee for their help in evaluating this paper.

\section{References}

Angelopoulos, V., Sibeck, D., Carlson, C. W., McFadden, J. P., Larson, D., Lin, R. P., Bonnell, J. W., Mozer, F. S., Ergun, R., Cully, C., Glassmeier, K. H., Auster, U., Roux, A., Le Contel, O., Frey, S., Phan, T., Mende, S., Frey, H., Donovan, E., Russell, C. T., Strangeway, R., Liu, J., Mann, I., Rae, J., Raeder, J., Li, X., Liu, W., Singer, H. J., Sergeev, V. A., Apatenkov, S., Parks, G., Fillingim, M., and Sigwarth, J.: The THEMIS Mission, Space Sci. Rev., 141, 5-34, doi:10.1007/s11214-008-9336-1, 2008a.

Angelopoulos, V., McFadden, J. P., Larson, D., Carlson, C. W., Mende, S. B., Frey, H., Phan, T., Sibeck, D. G., Glassmeier, K.-H., Auster, U., Donovan, E., Mann, I. R., Rae, I. J., Russell, C. T., Runov, A., Xhou, X., and Kepko, L.: Tail reconnection triggering substorm onset, Science, 321, 931-935, doi: 10.1126/science.1160495, 2008b.

Asano, Y., Mukai, T., Hoshino, M., Saito, Y., Hayakawa, H., and Nagai, T.: Current sheet structure around the near-Earth neutral line observed by Geotail, J. Geophys. Res., 109, A022212, doi: 10.1029/2003JA010114, 2004.

Attico, N., Califano, F., and Pegoraro, F.: Kinetic regimes of high frequency magnetic reconnection in a neutral sheet configuration, Phys. Plasmas, 9, 458-464, 2002.
Auster, H. U., Glassmeier, K. H., Magnes, W., Aydogar, O., Baumjohann, W., Constantinescu, D., Fischer, D., Fornacon, K. H., Georgescu, E., Harvey, P., Hillenmaier, O., Kroth, R., Ludlam, M., Narita, Y., Nakamura, R., Okrafka, K., Plaschke, F., Richter, I., Schwarzl, H., Stoll, B., Valavanoglou, A., and Wiedemann, M.: The THEMIS fluxgate magnetometer, Space Sci. Rev., 141, 135, doi:10.1007/s11214-008-9365-9, 2008.

Baumjohann, W., Roux, A., Le Contel, O., Nakamura, R., Birn, J., Hoshino, M., Lui, A. T. Y., Owen, C. J., Sauvaud, J.-A., Vaivads, A., Fontaine, D., and Runov, A.: Dynamics of thin current sheets: Cluster observations, Ann. Geophys., 25, 1365-1389, 2007, http://www.ann-geophys.net/25/1365/2007/.

Birn, J., Drake, J. F., Shay, M. A., Rogers, B. N., Denton, R. E., Hesse, M., Kuznetsova, M., Ma, Z. W., Battacharjee, A., Otto, A., and Pritchett, P. L.: Geospace Environmental Modeling (GEM) magnetic reconnection challenge, J. Geophys. Res., 106, 3715-3719, 2001.

Bonnell, J. W., Mozer, F. S., Delory, G. T., Hull, A. J., Ergun, R. E., Cully, C. M., and Angelopoulos, V.: The Electric Field Instrument (EFI) for THEMIS, Space Sci. Rev., 141, 303-341, doi:10.1007/s11214-008-9469-2, 2008.

Bulanov, S. V., Pegoraro, F., and Sakharov, A. S.: Magnetic reconnection in electron dynamics, Phys. Fluids B, 4, 2499-2508, 1992.

Chiu, Y. T. and Schulz, M.: Self-consistent particle and parallel electrostatic field distributions in the magnetopshericionospheric auroral region, J. Geophys. Res., 83, 629-642, 1978.

Cully, C. M., Ergun, R. E., Stevens, K., Nammari, A., and Westfall, J.: The THEMIS Digital Fields Board, Space Sci. Rev., 141, 343-355, doi:10.1007/s11214-008-9417-1, 2008.

Daughton, W., Lapenta, G., and Ricci, P.: Nonlinear evolution of the lower-hybrid drift instability in a current sheet, Phys. Rev. Lett., 93, 105004, doi:10.1103/PhysRevLett.93.105004, 2004.

Deng, X. H. and Matsumoto, H.: Rapid magnetic reconnection in the Earth's magnetosphere mediated by whistler waves, Nature, 410, 557-560, 2001.

Devine, P. E., Chapman, S. C., and Eastwood, J. W.: Oneand two-dimensional simulations of whistler mode waves in an anisotropic plasma, J. Geophys. Res., 100, 17189-17203, 1995.

Drake, J. F., Biskamp, D., and Zeiler, A.: Breakup of the electron current layer during 3-D collisionless magnetic reconnection, Geophys. Res. Lett., 24, 2921-2924, 1997.

Erickson, G. M., Maynard, N. C., Burke, W. J., Wilson, G. R., and Heinemann, M. A.: Electromagnetics of substorm onsets in the near-geosynchronous plasma sheet, J. Geophys. Res., 105, 25265-25290, 2000.

Gary, S. P.: Theory of space plasma microinstabilities, Cambridge atmospheric and space science serie, Cambridge university press, 1993.

Gary, S. P. and Karimabadi, H.: Linear theory of electron temperature anistropy instabilities: Whistler, mirror, and Weibel, J. Geophys. Res., 111, A11224, doi:10.1029/2006JA011764, 2006.

Gary, S. P. and Wang, J.: Whistler instability: Electron anisotropy upper bound, J. Geophys. Res., 101, 10749-10754, 1996.

Gurnett, D. A., Frank, L. A., and Lepping, R. P.: Plasma waves in the distant magnetotail, J. Geophys. Res., 81, 6059-6071, 1976.

Karimabadi, H., Daughton, W., and Quest, K. B.: Role of electron temperature anisotropy in the onset of magnetic reconnection, Geophys. Res. Lett., 31, L18801, doi:10.1029/2004GL020791, 
2004b.

Katoh, Y. and Omura, Y.: A study of generation mechanism of VLF triggered emission by self-consistent particle code, J. Geophys. Res., 111, A12207, doi:10.1029/2006JA011704, 2006.

Katoh, Y. and Omura, Y.: Computer simulation of chorus wave generation in the Earth's inner magnetosphere, Geophys. Res. Lett., 34, L03102, doi:10.1029/2006GL028594, 2007.

Kennel, C. F. and Petschek, H. E.: Limit on stably trapped particle fluxes, J. Geophys. Res., 71, 1, 1966.

Kennel, C. F., Coroniti, F. V., and Scarf, F. L.: Plasma waves in magnetotail flux ropes, J. Geophys. Res., 91, 1424-1438, 1986.

Kremser, G., Korth, A., Ullaland, S. L., Stadsnes, J., Baumjohann, W., Block, L., Torkar, K. M., Riedler, W., Aparicio, B., Tanskanen, P., Iversen, I. B., Cornilleau-Wehrlin, N., Solomon, J., and Amata, E.: Energetic electron precipitation during a magnetospheric substorm and its relationship to wave particle interaction, J. Geophys. Res., 91, 5711-5718, 1986.

Le Contel, O., Sahraoui, F., Roux, A., Fontaine, D., Robert, P., Sauvaud, J.-A., Owen, C., and Fazakerley, A. N.: Small scale Cluster observations of current sheet disruptions during substorms, in: Eighth International Conference on Substorms (ICS8), 27-31 March 2006, edited by: Syrjaeso, M. and Donovan, E., pp. 143-148, University of Calgary, Canada, 2006.

Le Contel, O., Roux, A., Robert, P., Coillot, C., Bouabdellah, A., de la Porte, B., Alison, D., Ruocco, S., Angelopoulos, V., Bromund, K., Chaston, C. C., and Cully, C.: First results of THEMIS Search Coil Magnetometers (SCM), Space Sci. Rev., 141, 509534, doi:10.1007/s11214-008-9371-y, 2008.

Li, W., Thorne, R. M., Angelopoulos, V., Bonnell, J. W., McFadden, J. P., Carlson, C. W., Mozer, F., Roux, A., Le Contel, O., Glassmeier, K. H., and Auster, U.: Evaluation of Chorus intensification during an injection event observed on THEMIS, J. Geophys. Res., 114, A00C14, doi:10.1029/2008JA013554, 2008.

Lui, A. T. Y., Angelopoulos, V., Mende, S. B., Le Contel, O., Frey, H., Donovan, E., Sibeck, D. G., Liu, W., Auster, H. U., Larson, D., Li, X., Nose, M., and Fillingim, M. O.: Determination of the substorm initiation region from a major conjunction interval of THEMIS satellites, J. Geophys. Res., 113, A00C04, doi:10.1029/2008JA013424, 2008.

Mandt, M. E., Denton, R. E., and Drake, J. F.: Transition to whistler mediated magnetic reconnection, Geophys. Res. Lett., 21, 7377, 1994.

McFadden, J. P., Carlson, C. W., Larson, D., and Angelopoulos, V.: THEMIS ESA first science results and performance issues, Space Sci. Rev., 141, 477-508, doi:10.1007/ s11214-008-9433-1, 2008.

Omura, Y., Katoh, Y., and Summers, D.: Theory and simulation of the generation of whistler-mode chorus, J. Geophys. Res., 113, A04223, doi:10.1029/2007JA012622, 2008.

Parrot, M., Santolk, O., Cornilleau-Wehrlin, N., Maksimovic, M., and Harvey, C.: Magnetospherically reflected chorus waves revealed by ray tracing with CLUSTER data, Ann. Geophys., 21, 1111-1120, 2003, http://www.ann-geophys.net/21/1111/2003/.

Passot, T., Ruban, V., and Sulem, P. L.: Fluid description of trains of stationary mirror structures in a magnetized plasma, Phys. Plasmas, 13, 102310, doi:10.1063/1.2356485, 2006.

Pellat, R., Coroniti, F. V., and Pritchett, P. L.: Does ion tearing exist?, Geophys. Res. Lett., 18, 143-146, 1991.

Perraut, S., Morane, A., Roux, A., Pedersen, A., Schmidt, R., Ko- rth, A., Kremser, G., Aparicio, B., and Pellinen, R.: Characterization of small scale turbulence observed at substorm onsets: Relationship with parallel acceleration of particles, Adv. Space Res., 13(4), 217-222, 1993.

Persson, H.: Electric field parallel to the magnetic field in a lowdensity plasma, Phys. Fluids, 9, 1090-1098, 1966.

Rönnmark, K.: WHAMP - Waves in homogeneous anisotropic multicomponent plasmas, Kiruna Geophysical Institute Report, 179, 1982.

Robert, P., Gendrin, R., Perraut, S., Roux, A., and Pedersen, A.: GEOS-2 identification of fastly moving current structures in the equatorial outer magnetosphere during substorms, J. Geophys. Res., 89, 819-840, 1984.

Rogers, B. N., Denton, R. E., Drake, J. F., and Shay, M. A.: Role of Dispersive Waves in Collisionless Magnetic Reconnection, Phys. Rev. Lett., 87, 195004, doi:10.1103/PhysRevLett.87.195004, 2001.

Roux, A. and Solomon, J.: Self-consistent solution of the quasilinear theory: Application to the spectral shape and intensity of VLF waves in the magnetosphere, J. Atmos. Terr. Phys., 33, 1457-1471, 1971.

Roux, A., Perraut, S., Robert, P., Morane, A., Pedersen, A., Korth, A., Kremser, G., Aparicio, B., Rodgers, D., and Pellinen, R.: Plasma sheet instability related to the westward traveling surge, 96, 17697-17714, 1991.

Roux, A., Le Contel, O., Robert, P., Coillot, C., Bouabdellah, A., la Porte, B., Alison, D., Ruocco, S., and Vassal, M. C.: The Search Coil Magnetometer (SCM) for THEMIS, Space Sci. Rev., 141, 265-275, doi:10.1007/s11214-008-9455-8, 2008.

Runov, A., Angelopoulos, V., Zhou, X., Voronkov, I., Kubyshkina, M., Nakamura, R., Carlson, C., Frey, H., McFadden, J., Larson, D. E., Mende, S., Glassmeier, K.-H., Auster, U., and Singer, H. J.: Multi-point in-situ and ground-based observations during auroral intensifications, J. Geophys. Res., 113, A00C07, doi:10.1029/JA2008013493, 2008.

Russell, C. T.: Noise in the geomagnetic tail, Planet. Space Sci., 20, 1541-1553, 1972.

Samson, J. C. and Olson, J. V.: Some comments on the descriptions of the polarization states of waves, Geophys. J. R. Astr. Soc., 61, 115-129, 1980.

Santolík, O., Gurnett, D. A., and Pickett, J. S.: Multipoint investigation of the source region of storm-time chorus, Ann. Geophys., 22, 2555-2563, 2004,

http://www.ann-geophys.net/22/2555/2004/.

Scarf, F. L., Coroniti, F. V., Kennel, C. F., Fredericks, R. W., Gurnett, D. A., and Smith, E. J.: ISEE 3 wave measurements in the distant geomagnetic tail and boundary layer, Geophys. Res. Lett., 11, 335-338, 1984.

Schindler, K. and Birn, J.: Models of two-dimensional embedded thin current sheets from Vlasov theory, J. Geophys. Res., 107, 1193, doi:10.1029/2001JA000304, 2002.

Shinohara, I., Nagai, T., Fujimoto, M., Terasawa, T., Mikai, T., Tsuruda, K., and Yamamoto, T.: Low-frequency electromagnetic turbulence observed near the substorm onset site, J. Geophys. Res., 103, 20365-20388, 1998.

Shiokawa, K., Miyashita, Y., Shinohara, I., and Matsuoka, A.: Decrease in $\mathrm{B}_{z}$ prior to the dipolarization in the near-Earth plasma sheet, J. Geophys. Res., 110, A09219, doi:10.1029/ 2005JA011144, 2005. 
Sigsbee, K., Cattell, C. A., Mozer, F. S., Tsuruda, K., and Kokubun, S.: Geotail observations of low-frequency waves from 0.001 to $16 \mathrm{~Hz}$ during the November 24, 1996, Geospace Environnement Modeling substorm challenge event, J. Geophys. Res., 106, 435445, 2001.

Sitnov, M., Malova, H., and Sharma, A.: Role of temperature ratio in the linear stability of the quasi-neutral sheet tearing mode, Geophys. Res. Lett., 25, 269-272, 1998.

Sitnov, M., Zelenyi, L. M., Malova, H. V., and Sharma, A.: Thin current sheet embedded within a thicker plasma sheet: Selfconsistent kinetic theory, J. Geophys. Res., 105, 13029-13043, 2000 .

Stenberg, G., Oscarsson, T., André, M., Vaivads, A., Morooka, M., Cornilleau-Wehrlin, N., Fazakerley, A., Lavraud, B., and Décréau, P. M. E.: Electron-scale sheets of whistlers close to the magnetopause, Ann. Geophys., 23, 3715-3725, 2005,

http://www.ann-geophys.net/23/3715/2005/.
Sydora, R. D., Sauer, K., and Silin, I.: Coherent whistler waves and oscilliton formation: Kinetic simulations, Geophys. Res. Lett., 34, L22105, doi:10.1029/2007GL031839, 2007.

Wei, X. H., Cao, J. B., Zhou, G. C., Santolík, O., Rème, H., Dandouras, I., Cornilleau-Wehrlin, N., Lucek, E., Carr, C. M., and Fazakerley, A.: Cluster observations of waves in the whistler frequency range associated with magnetic reconnection in the Earth's magnetotail, J. Geophys. Res., 112, A10225, doi:10. 1029/2006JA011771, 2007.

Zhang, Y., Matsumoto, H., and Kojima, H.: Whistler mode waves in the magnetotail, J. Geophys. Res., 104, 28633-28644, 1999. 